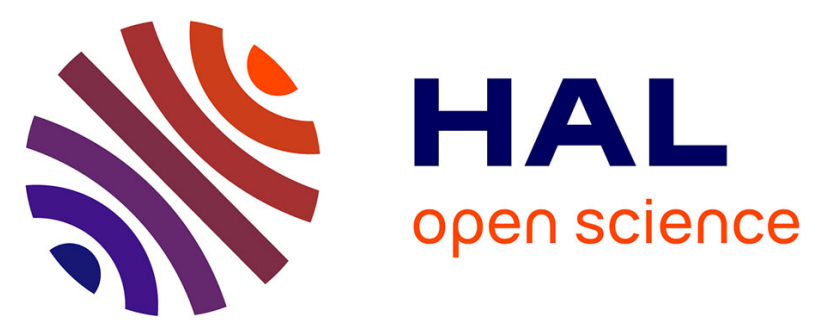

\title{
Exact analytic solutions of the porous media and the gas pressure diffusion ODEs in nonlinear mechanics
}

\author{
Dimitrios E. Panayotounakos, Anastasia B. Sotiropoulou, Nikos B.
}

Sotiropoulos, Manos Manios

\section{To cite this version:}

Dimitrios E. Panayotounakos, Anastasia B. Sotiropoulou, Nikos B. Sotiropoulos, Manos Manios. Exact analytic solutions of the porous media and the gas pressure diffusion ODEs in nonlinear mechanics. International Journal of Non-Linear Mechanics, 2007, 42 (1), pp.157. 10.1016/j.ijnonlinmec.2006.10.018 . hal-00501736

\section{HAL Id: hal-00501736 https://hal.science/hal-00501736}

Submitted on 12 Jul 2010

HAL is a multi-disciplinary open access archive for the deposit and dissemination of scientific research documents, whether they are published or not. The documents may come from teaching and research institutions in France or abroad, or from public or private research centers.
L'archive ouverte pluridisciplinaire HAL, est destinée au dépôt et à la diffusion de documents scientifiques de niveau recherche, publiés ou non, émanant des établissements d'enseignement et de recherche français ou étrangers, des laboratoires publics ou privés. 


\section{Author's Accepted Manuscript}

Exact analytic solutions of the porous media and the gas pressure diffusion ODEs in nonlinear mechanics

Dimitrios E. Panayotounakos, Anastasia B. Sotiropoulou, Nikos B. Sotiropoulos, Manos Manios

PII: $\quad$ S0020-7462(07)00031-5

DOI: doi:10.1016/j.ijnonlinmec.2006.10.018

Reference: NLM 1316

INTERNATIONAL JOURNAL OF

Reference:

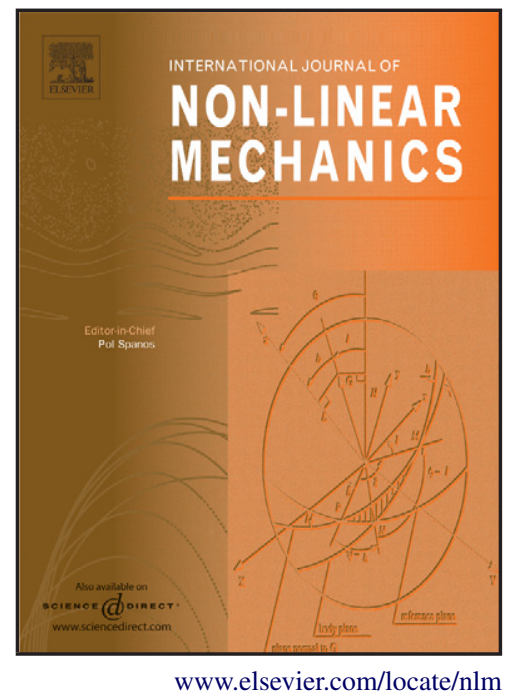

To appear in: International Journal of Non-

Linear Mechanics

Received date: 12 July 2006

Revised date: 2 October 2006

Accepted date: 18 October 2006

Cite this article as: Dimitrios E. Panayotounakos, Anastasia B. Sotiropoulou, Nikos B. Sotiropoulos and Manos Manios, Exact analytic solutions of the porous media and the gas pressure diffusion ODEs in nonlinear mechanics, International Journal of Non-Linear Mechanics (2007), doi:10.1016/j.ijnonlinmec.2006.10.018

This is a PDF file of an unedited manuscript that has been accepted for publication. As a service to our customers we are providing this early version of the manuscript. The manuscript will undergo copyediting, typesetting, and review of the resulting galley proof before it is published in its final citable form. Please note that during the production process errors may be discovered which could affect the content, and all legal disclaimers that apply to the journal pertain. 


\title{
Exact analytic solutions of the porous media and the gas pressure diffusion ODEs in nonlinear mechanics
}

\author{
Dimitrios E. Panayotounakos*, Anastasia B. Sotiropoulou, Nikos B. Sotiropoulos, \\ Manos Manios \\ National Technical University of Athens, Zographou Campus, \\ 5 Heroes of Polytechniou Avenue, Theocaris Building, GR 157 73,Athens, Hellas.
}

\begin{abstract}
Two kinds of second-order nonlinear ordinary differential equations (ODEs) appearing in mathematical physics and nonlinear mechanics are analyzed in this paper. The one concerns the Kidder equation in porous media and the second the gas pressure diffusion equation. Both these equations are strongly nonlinear including quadratic first order derivatives (damping terms). By a series of admissible functional transformations we reduce the prescribed equations to Abel's equations of the second kind of the normal form that they do not admit exact analytic solutions in terms of known (tabulated) functions. According to a mathematical methodology recently developed concerning the construction of exact analytic solutions of the above class of Abel's equations, we succeed in performing the exact analytic solutions of both Kidder's and gas pressure diffusion equations. The boundary and initial data being used in the above constructions are in according with each specific problem under considerations.
\end{abstract}

Keywords: Exact analytic solutions; Nonlinear ODEs; Kidder's equation; Gas pressure diffusion equation.

\section{Introduction}

The Kidder nonlinear ordinary differential equation (ODE) governs the problem of the unsteady flow of a gas through a semi-infinite porous medium [1, 2]. The origin of this equation is attractive, since it appears in the one dimensional problem obtained from the gas flow nonlinear partial differential equations. Until now, because of its strong nonlinearity (including quadratic first-order derivative damping term), only approximate or numerical solutions have been constructed under suitable boundary conditions. Furthermore, the equation itself consists a guideline for several relative problems appearing in the gas flow theory. In this paper using series of admissible functional transformations we reduce the Kidder nonlinear ODE to an equivalent Abel equation of the second kind of the normal form. It is well known [3, 4] that this class of equations do not admit exact analytic solutions in terms of known (tabulated) functions. Only very special types can be analytically solved in parametric form depending on the kind of its second free member [4].

A relative equation appearing in the theory of gas pressure problems is the onedimensional gas pressure diffusion equation [5]. We prove that this strongly nonlinear second-order ODE can be reduced to an equivalent generalized Emden-Fowler, or normal Emden-Fowler, or finally, to an Abel equation of the second kind of the normal form. Thus, according to the prescribed mathematical methodology, the construction of the exact analytic solution of this equation is obtained too.

The mathematical technique introduced is general and can be applied to a very large class of unsolvable (in terms of known tabulated functions) ODEs in nonlinear

\footnotetext{
* Corresponding author

e-mail address: tsakstel@cental.ntua.gr (D. E. Panayotounakos)
} 
mechanics and, generally, in mathematical physics (Blasius' eq.; Emden's eq.; Duffing's eq.; Van der Pol's eq.; Langmuir's eq., etc.)

\section{Preliminaries - Notation}

The equation due to R. F. Kidder [1] is the following

$$
\sqrt{1-a y} y_{x x}^{\prime \prime}+2 x y_{x}^{\prime}=0, \quad 0<a<1
$$

and appears in the problem of the unsteady flow of a gas through a semi-infinite porous medium. Here the notation $y_{x}^{\prime}=d y / d x, \quad y_{x x}^{\prime \prime}=d^{2} y / d x^{2} \ldots$ is used for total derivatives. The origin of the equation is attractive, since it appears in the one dimensional problem obtained from the gas flow nonlinear partial differential equation. The boundary conditions required by the physical problem are the following.

$$
\text { for } x=0, y(0)=1 \text { and for } x \rightarrow \infty, y(\infty) \rightarrow 0
$$

A semi analytical solution of the equation leading to expressions including the probability integral was constructed by means of the power series technique (see Ref. [2], pp. 410-411).

A similar to the above problem was recently provided in Ref. [5] and concerns the nonlinear gas-pressure diffusion equation appearing in the formulation of gas flow problems, where the fluid compressibility must be taken into account. This relative nonlinear ODE is of the type

$$
2 y y_{x x}^{\prime \prime}+5 y_{x}^{\prime 2}+x y_{x}^{\prime}=0
$$

with the following boundary conditions:

$$
\begin{aligned}
& \text { for } x \rightarrow \infty, \quad y=y_{\infty}=1, \text { and } \\
& \text { for } x=x_{0}=0, \quad 0<y_{0}<1 .
\end{aligned}
$$

In what follows we will prove that by a series of admissible functional transformations both Kidder's equation (2.1) and the gas pressure diffusion equation (2.3) can be reduced to several well-known nonlinear ODEs of the generalized Emden-Fowler's, or of the Emden-Fowler's, or finally, of Abel's types of equations. The prescribed equations do not admit exact analytic solutions in terms of known (tabulated) functions, since only very special cases of them can be analytically solved in parametric form (see Ref. [4], pp. 277-301; 241-250, and 29-55).

\section{The reduction procedure}

a) Kidder's equation

Consider the Kidder equation 


$$
\begin{gathered}
\sqrt{1-a y} y_{x x}^{\prime \prime}+2 x y_{x}^{\prime}=0, \quad 0<a<1, \\
\text { for } x=x_{0}=0, \quad y=y_{0}=1, \quad \text { and for } \\
x \rightarrow \infty, \quad y=y_{\infty}=0 .
\end{gathered}
$$

By the substitution

$$
1-a y=z(x),
$$

Eq. (3.1) results in the following generalized Emden-Fowler equation

$$
\begin{gathered}
z_{x x}^{\prime \prime}=-2 x z^{-1 / 2} z_{x}^{\prime} ; \quad\{n, m, l\}=\left\{1,-\frac{1}{2}, 1\right\}, \\
\text { for } x=x_{0}=0, \quad z=z_{0}=1-a, \text { and for } \\
x \rightarrow \infty, \quad z=z_{\infty}=1 .
\end{gathered}
$$

Here, we utilize the triad notation $\{n, m, l\}$ to denote the specific exponents of the reduced equation.

The F - transformation

$$
\mathrm{F}: \quad z_{x}^{\prime}=\frac{d z}{d x}=\frac{1}{\frac{d x}{d z}}=\frac{1}{x_{z}^{\prime}}
$$

reduces (3.3) into the alternative generalized Emden-Fowler equation

$$
\begin{gathered}
x_{z z}^{\prime \prime}=2 z^{-1 / 2} x\left(x_{z}^{\prime}\right)^{2} ; \quad\{n, m, l\}=\left\{-\frac{1}{2}, 1,2\right\}, \\
\text { for } z=1-a, \quad x=x_{0}=0, \text { and for } \\
z=z_{\infty}=1, \quad x \rightarrow \infty .
\end{gathered}
$$

Since in this case we have $n=-1 / 2 \neq-1, \quad m=1 \neq 0$ and $l=2 \neq 1$, the coordinate transformations (see Ref. [4], p. 300)

$$
\mathrm{S}: \quad w=z^{n+1}=z^{1 / 2}, \quad t=\left(x_{z}^{\prime}\right)^{1-l}=\left(x_{z}^{\prime}\right)^{-1},
$$

transform Eq. (3.5) to the generalized Emden-Fowler equation for $w=w(t)$ with the parameters changed:

$$
\begin{gathered}
w_{t t}^{\prime \prime}=-8 t^{-1} w\left(w_{t}^{\prime}\right)^{3} ; \quad\{n, m, l\}=\{-1,1,3\}, \\
\text { for } z=z_{0}=1-a, \quad w=w_{0}=\sqrt{1-a}, \text { and for } z=z_{\infty}=1, \quad w \rightarrow 1 .
\end{gathered}
$$

But, since $t_{w}^{\prime}=1 / w_{t}^{\prime}, \quad t_{w w}^{\prime \prime}=-w_{t t}^{\prime \prime} /\left(w_{t}^{\prime}\right)^{3}$, Eq. (3.7) becomes of the Emden-Fowler type

$$
t_{w w}^{\prime \prime}=8 w t^{-1} ; \quad\{n, m\}=\{1,-1\} \text {. }
$$


When the solution of the transformed equation (3.8) is obtained in the form of $t=t(w) \Rightarrow w=w(t)$, the solution to the original equation (3.5) can be written in the following parametric form. By the first transformation of (3.6) we have

$$
z=w^{2} \Rightarrow 1=2 w w_{z}^{\prime} \Rightarrow w_{z}^{\prime}=1 / 2 w
$$

while Eq. (3.5) can be rewritten as $\left(1 / x_{z}^{\prime}\right)_{z}^{\prime}=-2 x / \sqrt{z}$. Also, the second of (3.6) permits us to write the last equation as $t_{z}^{\prime}=-2 x / \sqrt{z}=t_{w}^{\prime} w_{z}^{\prime}=t^{\prime} / 2 w \Rightarrow x=-t_{w}^{\prime} / 4$.

Thus, the solution of Eq. (3.5) in parametric form results in

$$
z=w^{2}, \quad x=-\frac{1}{4}\left(w_{t}^{\prime}\right)^{-1}
$$

As we can see from the classification tables, concerning classes of second order nonlinear ODEs of Ref. [4], pp. 278-281; 242, none of the above generalized EmdenFowler, or of the normal form Emden-Fowler equations (3.3), (3.5), (3.7) and (3.8) admits an exact analytic solution.

In what follows it will be shown that both Emden-Fowler equations of the normal form (3.8) and generalized Emden-Fowler equation (3.5) can be reduced to Abel equations of the second kind of the normal form respectively. This can be achieved using convenient admissible functional transformations. We distinguish the following two cases.

Case a: The Emden-Fowler equation of the normal form (3.8).

Since the pair of indices $\{n, m\}$ of this equation satisfies, $m=-1 \neq 1$ and $m=1 \neq-2 n-3=-19 / 5$, there exists an admissible functional transformation that can reduce it to an Abel equation of the second kind of the normal form $y y_{x}^{\prime}-y=f(x)$ (see Ref. [4], p. 250). To show this we introduce the new variables $s$ and $u$ such that

$$
\mathrm{H}: \quad s=\frac{2 n+m+3}{m-1} w^{\frac{n+2}{m-1}} t, \quad u=w^{\frac{n+2}{m-1}}\left(w t_{w}^{\prime}+\frac{n+2}{m-1} t\right),
$$

that is to say such that

$$
\mathrm{H}: \quad s=-2 w^{-3 / 2} t, \quad u=w^{-3 / 2}\left(w t_{w}^{\prime}-\frac{3}{2} t\right),
$$

obtaining the following Abel equation

$$
u u_{s}^{\prime}-u=-\frac{3}{16} s-2 s^{-1}
$$

This Abel equation does not admit an exact analytic solution in parametric form (see Ref. [4], pp. 29-45).

Case b: The generalized Emden-Fowler equation (3.5).

The coordinate transformations 


$$
\mathrm{P}: \quad s=\frac{z}{x} x_{z}^{\prime}, \quad u=2 z^{-1 / 2} x^{2}
$$

reduce the first of Eqs. (3.5) to the following Abel equation of the second kind

$$
\left(s^{2} u-s^{2}+s\right) u_{s}^{\prime}=\left(2 s-\frac{1}{2}\right) u,
$$

which, by the substitution

$$
\stackrel{*}{u}=u-1+s^{-1}
$$

performs the simplified Abel equation of the second kind

$$
\stackrel{* *}{u u_{s}^{\prime}}=\frac{1}{s^{2}}\left(2 s-\frac{3}{2}\right) \dot{u}+\frac{1}{s^{2}}\left(2 s+\frac{1}{2 s}-\frac{5}{2}\right) .
$$

Finally, the well-known substitution ([4], p. 46)

$$
s=\int \frac{2 s-\frac{3}{2}}{s^{2}} d s=\ln s^{2}+\frac{2}{s^{3}}
$$

transforms (3.16), and thus (3.5), to the following Abel equation of the second kind of the normal form

$$
\stackrel{*}{* u_{s}^{\prime}}, u=\frac{(s-1)(4 s-1)}{s(4 s-3)}, \quad s=\ln s^{2}+\frac{2}{s^{3}} \text {. }
$$

Eq. (3.18) does not admit an exact solution in terms of known (tabulated) functions ([4], pp. 29-45). Both equations (3.12) and (3.18) are equivalent to the original Kidder equation (3.1) and their possible solutions constitute its intermediate integrals in the phase plane.

\section{b) The gas pressure diffusion equation}

To a similar to (3.5) generalized Emden-Fowler equation results also the nonlinear gas pressure diffusion equation (2.14). Indeed, the F - transformation (3.4)

$$
\mathrm{F}: \quad y_{x}^{\prime}=\frac{1}{x_{y}^{\prime}}
$$

transforms (2.3) to the following nonlinear ODE

$$
\begin{aligned}
& 2 y x_{y y}^{\prime \prime}-5 x_{y}^{\prime}-x x_{y}^{\prime 2}=0, \\
& \text { for } y=y_{\infty}=1, \quad x \rightarrow \infty \text {, and for } \\
& 0<y_{0}<1, \quad x=x_{0}=0 \text {. }
\end{aligned}
$$


Introducing the additional coordinate transformation

$$
x(y)=n(\xi), \quad \xi=\xi(x) \Rightarrow x_{y}^{\prime}=n_{\xi}^{\prime} \xi_{y}^{\prime}, \quad x_{y y}^{\prime}=n_{\xi \xi}^{\prime \prime} \xi_{y}^{\prime 2}+n_{\xi}^{\prime} \xi_{y y}^{\prime \prime}
$$

where $n$ and $\xi$ are to be determined, Eq. (3.20) becomes

$$
2 y \xi_{y}^{\prime 2} n_{\xi \xi}^{\prime \prime \prime}+\left(2 y \xi_{y y}^{\prime \prime}-5 \xi_{y}^{\prime}\right) n_{\xi}^{\prime}-\xi_{y}^{\prime 2} n n_{\xi}^{\prime 2}=0
$$

Specifying $\xi(y)$ such that

$$
2 y \xi_{y y}^{\prime \prime}-5 \xi_{y}^{\prime}=0 \Rightarrow \xi(y)=\frac{2}{7} y^{7 / 2}
$$

one extracts the following generalized Emden-Fowler equation for $n(\xi)$ with convenient initial and boundary data:

$$
\begin{gathered}
n_{\xi \xi}^{\prime \prime} "=A \xi^{-2 / 7} n n_{\xi}^{\prime 2}, \quad A=\frac{1}{2}\left(\frac{7}{2}\right)^{-2 / 7} ; \quad \xi=\frac{2}{7} y^{7 / 2}, \\
\text { for } n \rightarrow \infty, \quad \xi=\xi_{\infty}=\frac{2}{7} \text {, and for } n=n_{0}=0, \quad 0<\left(\frac{7}{2} \xi_{0}\right)^{2 / 7}<1 .
\end{gathered}
$$

An alternative form of (3.24) can be obtained by the already introduced F transformation. In fact, setting

$$
\mathrm{F}: \quad n_{\xi}^{\prime}=\frac{1}{\xi_{n}^{\prime}}
$$

we reduced (3.24) into the alternative generalized Emden-Fowler equation:

$$
\begin{gathered}
\xi_{n n}^{\prime \prime}=A n \xi^{-2 / 7} \xi_{n}^{\prime}, \quad A=\frac{1}{2}\left(\frac{7}{2}\right)^{-2 / 7} ; \\
\text { for } \xi=\xi_{\infty}=\frac{2}{7}, \quad n \rightarrow \infty, \quad \text { and for } n=n_{0}=0, \quad 0<\left(\frac{7}{2} \xi_{0}\right)^{2 / 7}<1 .
\end{gathered}
$$

Furthermore in equation (3.24) the exponents $n, m$ and $l$ of the variables $\xi, n$ and $n_{\xi}^{\prime}$, respectively follow the values: $n=-\frac{2}{7} \neq-1, \quad m=1 \neq 0$ and $l=2 \neq 1$. Thus the transformation [4, p. 300]

$$
\mathrm{S}: w(t)=\xi^{n+1}=\xi^{5 / 7}, \quad t=\left(n_{\xi}^{\prime}\right)^{1-l}=n_{\xi}^{\prime-1},
$$

transform (3.24) to the generalized Emden-Fowler equation

$$
w_{t t}^{\prime \prime}=B t^{-1} w^{2 / 5} w_{t}^{\prime 3}, \quad B=\frac{49}{25} A,
$$


which by way of the F -transformation, can be written to the Emden-Fowler equation of the normal form

$$
t_{w w}^{\prime \prime}=-B w^{2 / 5} t^{-1}, \quad B=\frac{49}{25} A
$$

with convenient boundary and initial data.

When the solution of the transformed equation (3.29) is obtained in the form $t=t(w) \Leftrightarrow w=w(t)$, the solution of the original equation (3.24) can be written in the parametric form

$$
\xi=w^{7 / 5}, \quad n=k\left(w_{t}^{\prime}\right)^{-1} ; \quad k=-\frac{5}{7 A} .
$$

In the next Section, according to the mathematical methodology recently developed in Refs.[6, 7], we will provide the exact analytic solution of the Kidder equation (2.1). Exactly the same mathematical technique follows the gas pressure diffusion equation (2.14).

\section{Exact analytic solution of the Kidder equation}

Consider the general Abel equation of the second kind of the normal form

$$
u u_{s}^{\prime}-u=f(s)
$$

where $f(s)$ is an arbitrary smooth function. In the present case (Eq. (3.12)), $f(s)$ is specified by the expression

$$
f(s)=A s+B s^{-1} ; \quad A=-\frac{3}{16}, \quad B=-2 .
$$

It was recently proved $[5,6]$ that the Abel equation (4.1) admits an exact analytic solution in terms of known (tabulated) functions. Summarizing the results of the above reference, we write the solution of the Abel equation (3.12) as follows:

$$
\begin{gathered}
u(s)=\frac{\left[\bar{N}(s)+\frac{1}{3}\right]\left(s+2 C_{1}\right)}{2}, \\
u_{s}^{\prime}=\frac{1}{2}\left[\bar{N}(s)+\frac{1}{3}\right]+\frac{2\left[G+2\left(A s+B s^{-1}\right)\right]}{\left(s+2 C_{1}\right)\left[\bar{N}(s)+\frac{4}{3}\right]}, \\
4\left[G+2\left(A s+B s^{-1}\right)\right]=-\frac{1}{4} \frac{\left[(\bar{\xi} \sin \bar{\xi}+\cos \bar{\xi})^{*} A+\cos ^{2} \bar{\xi}\right]\left(4 \bar{\xi}^{*} A+\cos \bar{\xi}\right)}{\left(\bar{\xi}^{*} A\right)^{3}} ;
\end{gathered}
$$




$$
\begin{gathered}
*^{*}=\operatorname{ci}(\bar{\xi})=\hat{\mathrm{A}}+\ln \bar{\xi}+\int_{0}^{\bar{\xi}} \frac{\sin t-1}{t} d t=\text { cosine integral } \\
\bar{\xi}=\ln \left|s+2 C_{1}\right| \Rightarrow d \bar{\xi}=\frac{1}{s+2 C_{1}} d x ; \\
\hat{\mathrm{A}}=0.5772156649015325 \ldots=\text { Euler's constant } \\
C_{1}=\text { integration constant }
\end{gathered}
$$

The function $\bar{N}(s)$ is given as in the following three cases:

Case a: $Q<0 \quad(p<0)$

$$
\begin{gathered}
\bar{N}^{(1)}(s)=2 \sqrt{-\frac{p}{3}} \cos \frac{a}{3}, \\
\bar{N}^{(2)}(s)=-2 \sqrt{-\frac{p}{3}} \cos \frac{a-\pi}{3}, \quad \bar{N}^{(3)}(s)=-2 \sqrt{-\frac{p}{3}} \cos \frac{a+\pi}{3} \\
\cos a=-\frac{q}{2 \sqrt{-\left(\frac{p}{3}\right)^{3}}}, \quad 0<a<\pi
\end{gathered}
$$

Case b: $Q>0$

$$
\bar{N}(s)=\sqrt[3]{-\frac{q}{2}+\sqrt{Q}}+\sqrt[3]{-\frac{q}{2}-\sqrt{Q}}
$$

Case c: $Q=0$

$$
\bar{N}^{(1)}(s)=2 \sqrt[3]{-\frac{q}{2}}, \quad \bar{N}^{(2)}(s)=\bar{N}^{(3)}(s)=-\sqrt[3]{-\frac{q}{2}}
$$

where:

$$
Q(s)=\frac{1}{27} p^{3}(s)+\frac{1}{4} q^{2}(s), \quad p(s)=-\frac{\mathrm{a}^{2}}{3}+b, \quad q(s)=\frac{2}{27} \mathrm{a}^{3}-\frac{1}{3} \mathrm{a} b+c,
$$

and

$$
\begin{gathered}
\mathrm{a}=-4, \quad b=\frac{3\left(s+2 C_{1}\right)+4 G(s)+4\left(A s+B s^{-1}\right)}{\left(s+2 C_{1}\right)}, \\
c=-\frac{4 G(s)+8\left(A s+B s^{-1}\right)}{\left(s+2 C_{1}\right)} .
\end{gathered}
$$


The subsidiary function $G(s)$ is determined by the second of Eqs. (4.3).

The already constructed analytic solutions defined by equations (4.3) to (4.8) constitute the intermediate integral (the solution in the phase plane) of the original Kidder equation (2.1). To show this one follows the inverse course through the already introduced admissible functional transformations. Thus, by the transformation (3.11), that reduces the Emden-Fowler equation (3.8) to the Abel equation of the normal form (3.12), we have the system of equations (3.11). This system, by means of Eqs. (4.3) to (4.8), can be equivalent written in the form:

$$
\begin{gathered}
w^{-1 / 2} t_{w}^{\prime}=f_{1}(s), \\
w^{-3 / 2} t=f_{2}(s) ; \\
f_{1}(s)=u+\frac{3}{4} s, \quad f_{2}(s)=-\frac{1}{2} s,
\end{gathered}
$$

where the expression $u=u(s)$ is given by Eq. (4.3). Dividing the two first of (4.9) between them and using the second of them, we obtain the equivalent functional relations:

$$
\begin{aligned}
& t_{w}^{\prime}=w^{1 / 2} f_{1}(s), \\
& t=w^{3 / 2} f_{2}(s)
\end{aligned}
$$

The second of these expressions furnishes

$$
d t=f_{2_{s}}^{\prime} w^{3 / 2} d s+\frac{3}{2} f_{2} w^{1 / 2} d w \Rightarrow t_{s}^{\prime}=w^{3 / 2} f_{2_{s}}^{\prime}+\frac{3}{2} w^{1 / 2} f_{2} w_{s}^{\prime},
$$

by means of which we estimate

$$
\begin{gathered}
t_{w}^{\prime}=t_{s}^{\prime} s_{w}^{\prime}=\frac{t_{s}^{\prime}}{w_{s}^{\prime}}=w^{1 / 2} f_{1} \Rightarrow t_{s}^{\prime}=f_{1} w^{1 / 2} w_{s}^{\prime}=w^{3 / 2} f_{2_{x}}^{\prime}+\frac{3}{2} w^{1 / 2} f_{2} w_{s}^{\prime} \Rightarrow \\
w_{s}^{\prime}=\frac{2 f_{2_{x}}^{\prime}}{2 f_{1}-3 f_{2}} w .
\end{gathered}
$$

Integrating we extract

$$
w=C_{2} \exp \left(-\int \frac{2 f_{2_{x}}^{\prime}}{2 f_{1}-3 f_{2}} d s\right)
$$

or, according to (4.9) we extract also

$$
w=C_{2} \exp \left(-\int \frac{d s}{2 u+3 s}\right),
$$

where $u(s)$ is the solution of the Abel equation (3.12) given by one of the expressions (4.4) to (4.6), and $C_{2}$ is a second constant of integration.

Summarizing, we list the following results: 
(i) Transformation H (Eqs. (3.11)):

$$
\begin{aligned}
& w=C_{2} \exp \left(-\int \frac{d s}{2 u+3 s}\right), \\
& t=w^{3 / 2} f_{2}=-\frac{1}{2} s C_{2} \exp \left(-\int \frac{d s}{2 u+3 s}\right), \\
& t_{w}^{\prime}=\frac{1}{w_{t}^{\prime}}=w^{1 / 2} f_{1}=\left(u+\frac{3}{4} s\right) C_{2} \exp \left(-\int \frac{d s}{2 u+3 s}\right) ;
\end{aligned}
$$

(ii) Transformation F (Eqs. (3.7)):

$$
w_{t}^{\prime}=\frac{1}{t_{w}^{\prime}}=\frac{C_{2}^{-1}}{\left(u+\frac{3}{4} s\right)} \exp \left(\int \frac{d s}{2 u+3 s}\right)
$$

(iii) Transformation S (Eqs. (3.6) and (3.9)):

$$
\begin{aligned}
& z=w^{2}=C_{2}^{2} \exp \left(-2 \int \frac{d s}{2 u+3 s}\right), \\
& x_{z}^{\prime}=\frac{1}{t}=-2 s^{-1} C_{2}^{-1} \exp ^{-1}\left(-\int \frac{d s}{2 u+3 s}\right) \\
& x=-\frac{1}{4} w_{t}^{\prime}=-\left(u+\frac{3}{4} s\right)^{-1} C_{2} \exp \left(\int \frac{d s}{2 u+3 s}\right) / 4
\end{aligned}
$$

(iv) Transformation (3.2) - Final solution:

$$
\begin{aligned}
y= & \frac{1-z}{a}=\frac{1}{a}\left[1-C_{2}^{2} \exp \left(-2 \int \frac{d s}{2 u+3 s}\right)\right], \\
x= & -\left(u+\frac{3}{4} s\right)^{-1} C_{2} \exp ^{-1}\left(\int \frac{d s}{2 u+3 s}\right) / 4 ; \\
u= & u(s)=\text { solution of the Abel equation (3.12) including } \\
& \text { one constant of integration } C_{1} ; \\
C_{2}= & \text { second indegration costant } ; \\
s= & \text { parameter . }
\end{aligned}
$$

The final results for the gas pressure diffusion equation (2.3), that correspond to the final solution (4.18) concerning the Kidder equation (2.1), are the following: 


$$
\begin{aligned}
& y=\left(\frac{7}{2}\right)^{2 / 7}\left[\exp \left(-\int \frac{d s}{u}\right)\right]^{2 / 7}, \\
& x=\frac{7}{5} \int \frac{1}{s u}\left[\exp \left(-\frac{6}{7} \int \frac{d s}{u}\right)\right]^{-1}\left[\exp \left(-\int \frac{d s}{u}\right)\right] d s+C_{2},
\end{aligned}
$$

where $u=u(s)$ is the solution of the Abel equation

$$
u u_{s}^{\prime}-u=A s+B s^{-1}
$$

with

$$
A=-\frac{6}{49}, \quad B=-\frac{1}{2}\left(\frac{7}{2}\right)^{-2 / 7}
$$

We underline that because of the various types that the function $\bar{N}(s)$ admits (formulae (4.4) to (4.6)), it is possible that the solutions of both Kidder's and gas pressure diffusion equations are not unique inside a main interval $\left[s_{0}, s_{F}\right]$; in other words, they can be divided into several branches of solutions valid separately inside convenient consecutive subintervals. In this case matching of the corresponding solutions must be performed in all values of the main interval that solutions change. In what follows, using the results of previous sections, we provide a solution technique concerning the boundary value problem of the Kidder equation.

\section{Boundary value problem - Solution technique}

The boundary conditions and the solution technique that follows concern the Kidder equation (2.1). Similar procedure one also follows for the gas pressure diffusion equation (2.3).

The boundary conditions of the problem under consideration have been already prescribed in Section 2, that is:

$$
\begin{gathered}
\text { for } x=x_{0}=0, \quad y=y_{0}=1, \quad \text { and } \\
\text { for } x \rightarrow \infty \quad y=y_{\infty}=0 .
\end{gathered}
$$

According to the substitution (3.2) the above boundary data become

$$
\text { for } \begin{gathered}
x=x_{0}=0, \quad z=z_{0}=1-a, \text { and for } \\
x=x_{\infty}=\infty, \quad z=z_{\infty}=1,
\end{gathered}
$$

while according to the formulae (3.9) become also

$$
\begin{gathered}
\text { for } z=z_{0}=1-a, \quad w=w_{0}=\sqrt{1-a} \\
w_{t_{0}}^{\prime}=-\frac{1}{4 x_{0}}=\infty, \quad \text { and for } z=z_{0}=1, \\
w=w_{\infty}=1 ; \quad w_{t_{\infty}}^{\prime}=-\frac{1}{4 x_{\infty}}=0 .
\end{gathered}
$$


The first set of Eqs. (5.3) will be used for the evaluation of the intermediate integral in the phase plane, while the second set for the calculation of the final solution in the physical plane.

Thus, combining the first set of the boundary data (5.3) together with the coordinate transformations (3.11), we extract

$$
\begin{gathered}
s_{0}=-\frac{2}{(1-a)^{3 / 2}} t_{0}, \\
u_{0}=\frac{1}{(1-a)^{3 / 2}}\left(\frac{w_{0}}{w_{t_{0}}^{\prime}}-\frac{3}{2} t_{0}\right)=-\frac{3 t_{0}}{2(1-a)^{3 / 2}} \Rightarrow s_{0}=\frac{4 u_{0}}{3} .
\end{gathered}
$$

Consequently, the Abel equation (4.2) and the solution (4.3) result in the following four equations:

$$
\begin{gathered}
u_{0}\left(u_{s_{0}}^{\prime}-1\right)=A s_{0}+B s_{0}^{-1}=\frac{4 A}{3} u_{0}+\frac{3 B}{4} u_{0}^{-1}, \\
u_{0}=\frac{1}{2}\left(\bar{N}_{0}+\frac{1}{3}\right)\left(\frac{4 u_{0}}{3}+2 C_{1}\right), \\
4\left(G_{0}+\frac{8 A u_{0}}{3}+\frac{6 B}{4 u_{0}}\right)=\frac{1}{4} \frac{\left[\left(\bar{\xi}_{0} \sin \bar{\xi}_{0}+\cos \bar{\xi}_{0}\right) A_{0}+\cos ^{2} \bar{\xi}_{0}\right]\left(4 A \bar{\xi}_{0} A_{0}+\cos \bar{\xi}_{0}\right)}{\left.\left(\bar{\xi}_{0} A_{0}\right)^{3}\right)} \\
\bar{\xi}_{0}=\ln \left|\frac{4 u_{0}}{3}+2 C_{1}\right|, \bar{A}_{0}=\operatorname{ci}\left(\bar{\xi}_{0}\right), \\
u_{s_{0}}^{\prime}=\frac{1}{\bar{N}_{0}+\frac{4}{3}} \frac{2\left[G_{0}+2\left(\frac{4 A}{3} u_{0}+\frac{3 B}{4} u_{0}^{-1}\right)\right]}{s_{0}+2 C_{1}}+\frac{1}{2}\left(\bar{N}_{0}+\frac{1}{3}\right)
\end{gathered}
$$

where $\bar{N}_{0}$ is the value of $\bar{N}(s)$ at $s=s_{0}$, and $G_{0}$ is the value of the subsidiary function $G(s)$ included in Eqs. (4.7), which is to be determined.

The set of the four equations (5.5) include five unknowns $u_{0}, u_{s_{0}}^{\prime}, \bar{N}_{0}, G_{0}$ and $C_{1}$. Thus, one more equation is needed for the estimation of the above five unknowns. This equation can be derived by means of the coordinate transformations (3.11). Indeed, by way of (3.11) and (3.8) we evaluate

$$
\begin{aligned}
& u_{s}^{\prime}=\frac{u_{t}^{\prime}}{s_{t}^{\prime}}, \quad s_{t}^{\prime}=\left[-3 w^{-5 / 2} t-2 w^{-3 / 2}\left(w_{t}^{\prime}\right)^{-1}\right] w_{t}^{\prime}, \\
& u_{t}^{\prime}=\left[\frac{2}{4} w^{-5 / 2} t+w^{-7 / 2} t-2 w^{-3 / 2}\left(w_{t}^{\prime}\right)^{-1}\right] w_{t}^{\prime} .
\end{aligned}
$$

Thus, since $w_{t_{0}}^{\prime}=\infty ; \quad w_{0}=\sqrt{1-a} ; \quad t_{0}=-(1-a)^{3 / 2} s_{0} / 2=-2(1-a)^{3 / 2} u_{0} / 3$, we extract 


$$
u_{s_{0}}^{\prime}=\frac{u_{t_{0}}^{\prime}}{s_{t_{0}}^{\prime}}=-\frac{3 w_{0}^{-5 / 2} t_{0}+2 w_{0}^{-3 / 2}}{\frac{9}{4} w_{0}^{-5 / 2} t_{0}+w_{0}^{-7 / 2} t_{0}}=\frac{12(1-a)^{1 / 2}\left[1-(1-a) u_{0}\right]}{\left[9+4(1-a)^{-1 / 2}\right] u_{0}} .
$$

Equations (5.5) and (5.7) constitute a nonlinear (transcendental) system by the solution of which one estimates $u_{0}, u_{s_{0}}^{\prime}, \bar{N}_{0}, G_{0}$ and $C_{1}$ in terms of the initial parameters. Therefore, since $\bar{N}_{0}$ is known, the discriminant $Q(s)$ defined in (4.7) is also known, fact that permits us to define the type of the solution $\bar{N}_{0}(s)$ valid inside the first subinterval $\left[s_{0}, s_{1}\right]$ of the main interval $\left[s_{0}, s_{F}\right]$. At the unknown point $\left(s_{1}, s_{2}\right]$, where the solution probably changes, we provide the following equations (the upper index in the parenthesis denotes the corresponding subinterval $\left.{ }^{(i)} ; \quad i=0,1,2, \ldots\right)$ :

(i) Common solution

$$
\left(\bar{N}_{s_{1}}^{(1)}+\frac{1}{3}\right)\left(s_{1}+2 C_{1}^{(1)}\right)=\left(\bar{N}_{s_{1}}^{(0)}+\frac{1}{3}\right)\left(s_{1}+2 C_{1}^{(0)}\right)
$$

(ii) Common derivative

$$
\left(\bar{N}_{s_{1}}^{(1)}+\frac{1}{3}\right)+\frac{2\left(G_{s_{1}}^{(1)}+A s_{1}+B s_{1}^{-1}\right)}{\left(s_{1}+2 C_{1}^{(1)}\right)\left(\bar{N}_{1}^{(1)}+\frac{4}{3}\right)}=\left(\bar{N}_{s_{1}}^{(0)}+\frac{1}{3}\right)+\frac{2\left(G_{s_{1}}^{(0)}+A s_{1}+B s_{1}^{-1}\right)}{\left(s_{1}+2 C_{1}^{(0)}\right)\left(\bar{N}_{1}^{(0)}+\frac{4}{3}\right)}
$$

(iii) Validity of the third of Eqs. (5.5)

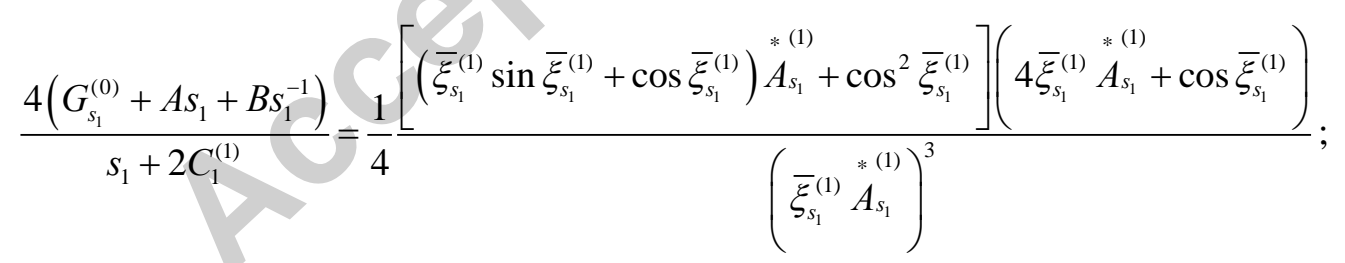

$$
\begin{aligned}
& \stackrel{*(1)}{A_{s_{1}}}=\operatorname{ci}\left(\bar{\xi}_{s_{1}}^{(1)}\right)=\text { cosine integral }, \bar{\xi}_{s_{1}}^{(1)}=\ln \left|s_{1}+2 C_{1}^{(1)}\right|
\end{aligned}
$$

(iv) Validity of the Cardan equation

$$
\bar{N}_{s_{1}}^{3(1)}+p_{s_{1}}^{(1)} \bar{N}_{s_{1}}^{(1)}+q_{s_{1}}^{(1)}=0,
$$

where $\bar{N}_{s_{1}}^{(1)}, \quad p_{s_{1}}^{(1)}, \quad q_{s_{1}}^{(1)}$ are calculated through Eqs. (4.4) to (4.7).

The nonlinear (transcendental) system of Eqs. (5.8) to (5.11) enables us to estimate $s_{1}, \bar{N}_{s_{1}}^{(1)}, G_{s_{1}}^{(1)}$ and $C_{1}^{(1)}$ in terms of the initial parameters as well as of the known 
parameters $\bar{N}_{s_{1}}^{(0)}, G_{s_{1}}^{(0)}$ and $C_{1}^{(0)}$. Thus, at the point $\left(s_{1}, s_{2}\right)$, where the solution probably changes, and for the consecutive subinterval $\left(s_{1}, s_{2}\right], Q_{s_{1}}^{(1)}$ given in Eqs. (4.7) is known. This, enables us to define the type of the solution valid inside $\left(s_{1}, s_{2}\right]$ (Eqs. (4.4) to (4.6)).

The prescribed analysis demands successive solutions of nonlinear (transcendental) systems which are must be satisfied for each of the above mentioned consecutive subintervals $\left(s_{i}, s_{i+1}\right]$, where the solution of the problem under consideration (in the phase plane) probably changes according to the formulae (4.4) to (4.6). From now on, the solution in the physical plane is obtained through the formulae (4.18).

\section{Discussion and conclusions}

By a series of admissible functional transformations we reduce the nonlinear porous media and gas pressure diffusion ODEs to Abel's equations of the second kind of the normal form. These equations do not admit exact analytic solutions in terms of known (tabulated) functions. This unsolvability is due to the fact that only very special forms of this kind of equation can be solved in parametric form [4]. Our goal is the development of the construction of exact analytic solutions of the above equations based on a mathematical technique leading to the derivation of exact analytic solutions of the Abel equation of the second kind of the normal form (see Refs. [6], [7]).

The reduction procedure introduced in the paper and the constructed solutions are very general, and can be applied to a large number of nonlinear ODEs in mathematical physics and nonlinear mechanics including the Van der Pol nonlinear oscillator, the Blasius equation in fluids [7], the Langmuir equation in current flow, etc.

For verification of the above contentions, we shall examine briefly one of the prescribed equations and compare its solution methodology with the already developed solutions procedure.

The Van der Pol free nonlinear oscillator [2] is governed by the following nonlinear ODE

$$
y_{x x}^{\prime \prime}-\varepsilon\left(1-y^{2}\right) y_{x}^{\prime}+y=0 ;-\infty<x<+\infty
$$

where $\varepsilon$ is a real positive parameter. By the substitution

$$
y_{x}^{\prime}=\omega(y) \Rightarrow y_{x x}^{\prime \prime}=\omega_{y}^{\prime} y_{x}^{\prime}=\omega \omega_{y}^{\prime}
$$

Eq. (6.1) is reduced to the following Abel equation of the second kind:

$$
\omega \omega_{y}^{\prime}-\varepsilon\left(1-y^{2}\right) \omega+y=0, \quad-\infty<y<\infty .
$$

Introducing the coordinate transformations

$$
\omega(y)=\omega(s), \quad s=\varepsilon y-\varepsilon \frac{y^{3}}{3}
$$


we obtain the Abel equation of the second kind of the normal form

$$
\omega \omega_{s}^{\prime}-\omega=-\frac{1}{\varepsilon} \frac{y}{1-y}, \quad y^{3}-3 y+\frac{3}{\varepsilon} s=0 ; \quad \varepsilon>0 ;-\infty<s<\infty, \quad-\infty<x<\infty
$$

Eqs. (6.5) are of the Abel normal form and they are similar to the Kidder and the gas pressure diffusion equations with different right-hand sides. With a set of similar or boundary initial conditions as in the before mentioned cases, the mathematical methodology for the exact analytic solutions of the above equations follows step by step that prescribed in Sections 3 and 4. 


\section{References}

[1] N. E. Kidder, Unsteady flow of gas through a semi-infinite porous medium, $J$. Appl. Mech. ASME 27 (1957) 329-332.

[2] H. T. Davis, Introduction to Nonlinear Differential and Integral Equations, Dover Publ., Inc., New York, 1962.

[3] E. Kamke, Differentialgleichungen, Lösungsmethoden und Lösungen, Vol. 1, B. G. Teubner, Stuttgard, 1977.

[4] A. D. Polyanin, V. F. Zaitsev, Handbook of Exact Solutions for Ordinary Differential Equations, CRC Press, Boca Raton, New York, London, Tokyo, 1995.

[5] I. Vardoulakis, J. Sulem, Bifurcation Analysis in Geomechanics, Blackie Academic and Professional, 1995.

[6] D. E. Panayotounakos, Exact analytic solutions of unsolvable classes of first and second order nonlinear ODEs (Part I: Abel's equations), Appl. Math. Letters 18 (2005) 155-162.

[7] D. E. Panayotounakos, N. B. Sotiropoulos, Exact analytic solutions of nonlinear boundary value problems in fluid mechanics (Blasius equation), J. of Math.

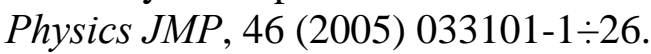

